\title{
Steering Wheel Control in Lane Departure Warning System
}

\author{
Maximiliano Hernández García Rojas ${ }^{1}$, Humberto Velasco Arellano ${ }^{1}$, \\ David Ubach González ${ }^{2}$, Martín Montes Rivera ${ }^{1}$, Marving Omar Aguilar Justo ${ }^{3}$ \\ ${ }^{1}$ Universidad Politécnica de Aguascalientes, Ciencias en Ingeniería, Aguascalientes, \\ Mexico \\ ${ }^{2}$ Universidad Politécnica de Aguascalientes, Ingeniería en Electrónica, Aguascalientes, \\ Mexico \\ ${ }^{3}$ Universidad Veracruzana, Veracruz, \\ Mexico \\ \{mc160006, mc150008, up140172\}@alumnos.upa.edu.mx, \\ martin.montes@upa.edu.mx,marvaguilar@uv.mx
}

\begin{abstract}
Advanced driver assistance systems (ADAS) are important to prevent road accidents, one of those are lane invasions. Nowadays optical and control systems are applied in new cars to maintain vehicles centered in a lane, nevertheless old vehicles cannot access to these new assistant systems. In this paper we present a position steering wheel control (SWC) in a lane detection prototype designed for its implementation in cars that do not have installed this system. The proposed technique uses a self-tuning PID controller connected to a DC motor for SWC that uses orientation of lines on the road. Lines are detected by applying Sobel filter convolution and Hough transform, which are image processing techniques that allow obtaining orientation. The PID controller is automatically tuned since this assistant would be installed in different cars. GA is used since it has shown good results parametrizing well known structured problems without requiring mathematical models or techniques that demand control experts. The main contributions of this research are a SCW alternative capable of been installed in any old vehicle and the algorithm implemented for using orientation as input data as the control reference with a self-tuning PID controller.
\end{abstract}

Keywords: steering wheel control, genetic algorithm, advanced driver assistance systems.

\section{Introduction}

In the car industry, one of the most important issues is user safety. In order to reduce current accidents and protecting the life of drivers, researchers have been looking for 
driver assistants. The most advance of them evaluate the traffic situations, using sensors or cameras, and support the driver to avoid damages [16].

The research for improving intelligent vehicles using advanced driver assistance systems (ADAS), includes the lane departure warning (LDW) system, which alarms the driver when the car is out of the line markings and apply the brakes to help him to move the vehicle [23].

It took the tool of LDW to be applied in not manned vehicles to control the direction and management across the real world to improve the vehicle interaction, for this reason was important to guaranty a position steering wheel control (SWC), to modify the vehicle trajectory closing the control loop in the LDW.

In this work, we propose an SWC based in orientation of lines on the road, where an automatic PID controller is tuned by a Genetic Algorithm (GA) that adapts the controller to any vehicle without deep knowledge in the mathematical background.

Self-tuning PID controller is required, because of the variation when changing different vehicles, is here where de adaptability and evolution in a GA allows getting a tuning method for any car [20].

The selection of a GA as tuning method it is the advantage that represents at the moment to find results in an optimization problem, where the solution presents a nonlinear behavior and in a multi-objective solution, gives a robustness method [20].

\section{$2 \quad$ Related Works}

In the last years, the LDW has been investigated, and some important contributions explored the safety field in science, like the investigation in [15], reporting comparison between the line detection system in a virtual video and a real video which proved that the real video and the natural noise increase the effectiveness in detection.

In 2015 was report a comparison between two different ways of alarm in LDW, one activating the breaker and the other modifying the steering wheel position, they discussed that the difference in simulated experiments was no profitable [4], at the time was propose the LDW using Hough transform, reporting a 3\% of false alarm, reducing the standard in that year [10]. In the following years the investigation focus in use of different filters [16, 23, 14], the application of observers [7, 21] and effectiveness evaluations [24], but in all cases, the application of LDW is just for alarm and to our best knowledge is no suitable for non-manned vehicles, on the other hand, the SWC patents describe the application and uses [1, 11, 13], where the application most significant is in a truck where was consider velocity of reaction to avoid insures [12], the review in [3], supports that non-manned vehicles have enough study with this technique.

Other works focuses on testing different lines filtering or edge detection techniques, as well as different types of line identification techniques $[8,13,14]$, but only generates an alert to the driver as an output.

Works such as $[1,3,11]$, use automatic steering wheel control systems on vehicles, but these are applied on modified vehicles and cannot be applied on other vehicles without a modification. 
In this paper we have included a way that helps to optimize the control processes with genetic algorithm, this is proposed because this technique has presented important contributions in diverse areas [18], for example: reduction of electric circuits [6], recovering of unknown parameters in oscillators [9], multi criteria decision taking [22], and in the control area, has been show important results like the use of a genetic algorithm for obtaining the PID parameters of a electrohydraulic servo control system [2].

The research in [17], follows the optimization of PI gains in a controller and compares the differences between the results of a traditional genetic algorithm and a proposed micro genetic algorithm.

\section{Theoretical Framework}

Control systems are used to maintain process conditions at their desired values by manipulating certain process variables to adjust the interest variables [5]. Tuning method is an indispensable step, when the controller is adjusted to have a required behavior for the interest variable, there are several methods for adjusting the conventional PID controller but in this paper the Ziegler and Nichols method will be used, since it is one of the most popular tuning methods [19].

\subsection{Ziegler and Nichols}

Ziegler and Nichols proposed some tuning rules for PID controller based on an experimental response, their main objective was to adjust the controllers without knowledge of the mathematical representation of the systems [19]. The result was the definition of two methods, in this case, it is explained the ultimate gain because it will be compare with the results achieved with a GA.

\subsection{Ultimate Gain}

This method is used when the system presents a second or greater order response, in that case the PID controller parameters are set for eliminating the integrative and derivative effects, then the proportional gain is increased in order to generate a sustain oscillation, at this point the value of gain is named critical gain, and de period that exists in the oscillation is name critical period. Then the P, I and D gains are calculated as in Table 1.

Table 1. Tuning values for ultimate gain method.

\begin{tabular}{ccccc}
\hline Controller & & $k_{p}$ & $\tau_{i}$ & $\tau_{d}$ \\
\hline $\mathrm{P}$ & 0.5 & $K_{c r}$ & $\infty$ & 0 \\
\hline $\mathrm{PI}$ & 0.45 & $K_{c r}$ & $0.5 P_{c r}$ & 0 \\
\hline $\mathrm{PID}$ & 0.6 & $K_{c r}$ & $0.5 P_{c r}$ & $0.125 P_{c r}$ \\
\hline
\end{tabular}




\subsection{Digital Control}

The control application is a studied science with too many branches, one of those is the digital control, and it has a big impact at moment to use digital circuits in computing, just hire the characteristics of this control need an indispensable a well defined sample period to guaranty the constant function, because it does not matter if the system works as fast as possible, the behavior is no continued [8].

The important advantages of digital control are the high flexibility and the capability to control a greater number of systems and optimize the time and space that is consumed by the controller, but it represents a higher price and is required special knowledge to manipulate them [19]. A general block diagram of a digital control loop is shown in Fig. 1, where is possible to see the transformation of the signals to be modified by a digital controller.

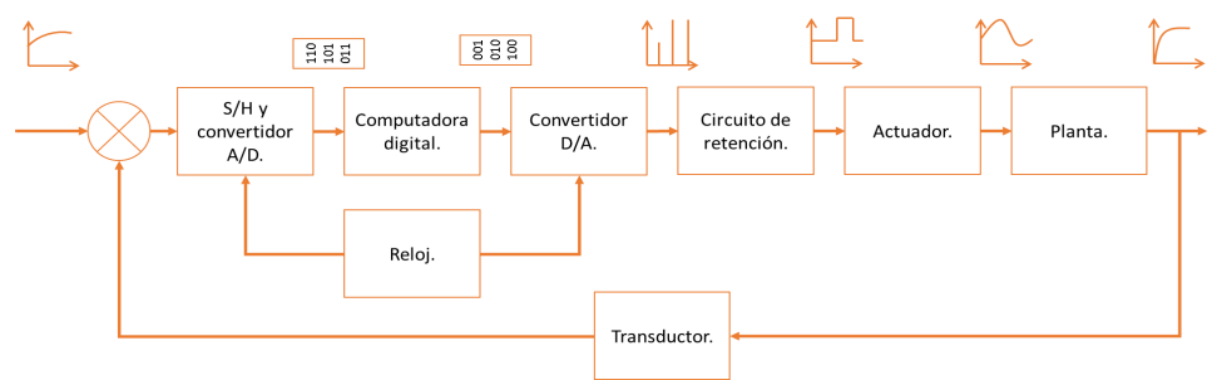

Fig. 1. Digital control close loop [16].

\subsection{Genetic Algorithm}

Evolutionary algorithms are population-based metaheuristic optimization algorithms that use biology-inspired mechanisms, in order to refine a set of solution candidates iteratively, the cycle of evolutionary algorithms follows the Darwin laws that are described in Fig. 2 [25].

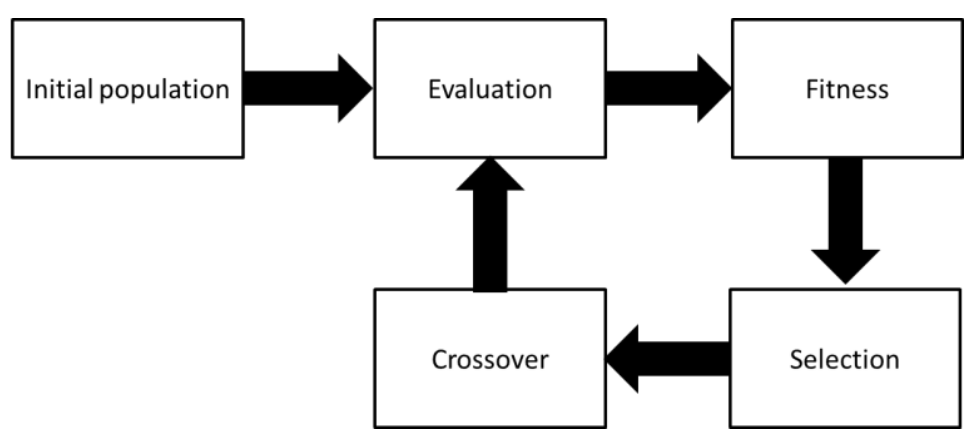

Fig. 2. Evolutionary Algorithms behavior. 
As is shown in Fig. 2, the operations in a genetic algorithm have a defined process, first the initial population is random initialized for obtaining a high diversity of possible solutions, the evaluation consists in the analysis of each individual by submitting it in the process, in order to measure its quality for solving the problem. The fitness is the assignation of a numerical value depending on the behavior presented. The selection is a process where 2 individuals are selected according to its fitness and finally, the crossover take that 2 individuals to generate new members combining their genes [25].

\section{Methodology}

The methodology explains how it is implemented the control to get important results that could contribute measuring the differences between a conventional controller and a GA tuning method to guaranty the control and its adaptation in different cars for the SWC and the LDW systems.

\subsection{Control}

Ziegler and Nichols tuning control method is applied in the digital PID controller described in equation 1 :

$$
U(z)=K p\left[1+\frac{T}{t i(1-z-1)}+\frac{t d\left(1-z^{-1}\right)}{T}\right],
$$

which is represented as the transfer function for the GA like in equation 2:

$$
\frac{U(z)}{E(z)}=a+\frac{b}{1-z-1}+c\left(1-z^{-1}\right)
$$

where the auxiliary variables are described in equation 3 :

$$
a=K p, \quad b=\frac{K p T}{t i}, c=\frac{K p t d}{T}
$$

The input signal is the error between reference angle and the position angle of the motor, and the output is the duty cycle of a PWM that rotates the motor.

To determine the rotation direction of the motor, the sign of the output is used, if negative then turns to the left and if positive it turns to the right. Then the absolute value is used as duty for a PWM output.

Once the controller structure it is built, it is necessary to adjust the gains. For this propose the Ziegler-Nichols method of critical gain for systems with sustained oscillations was used. This method allows obtaining the gains $k_{p}, \tau_{i}$ and $\tau_{d}$ for the PID controller without knowing the model of the plant.

First, the integral and derivative part are eliminated, leaving a proportional controller. The $k_{p}$ gain of this controller is modified until the system oscillates steadily as shown in Fig. 3. 


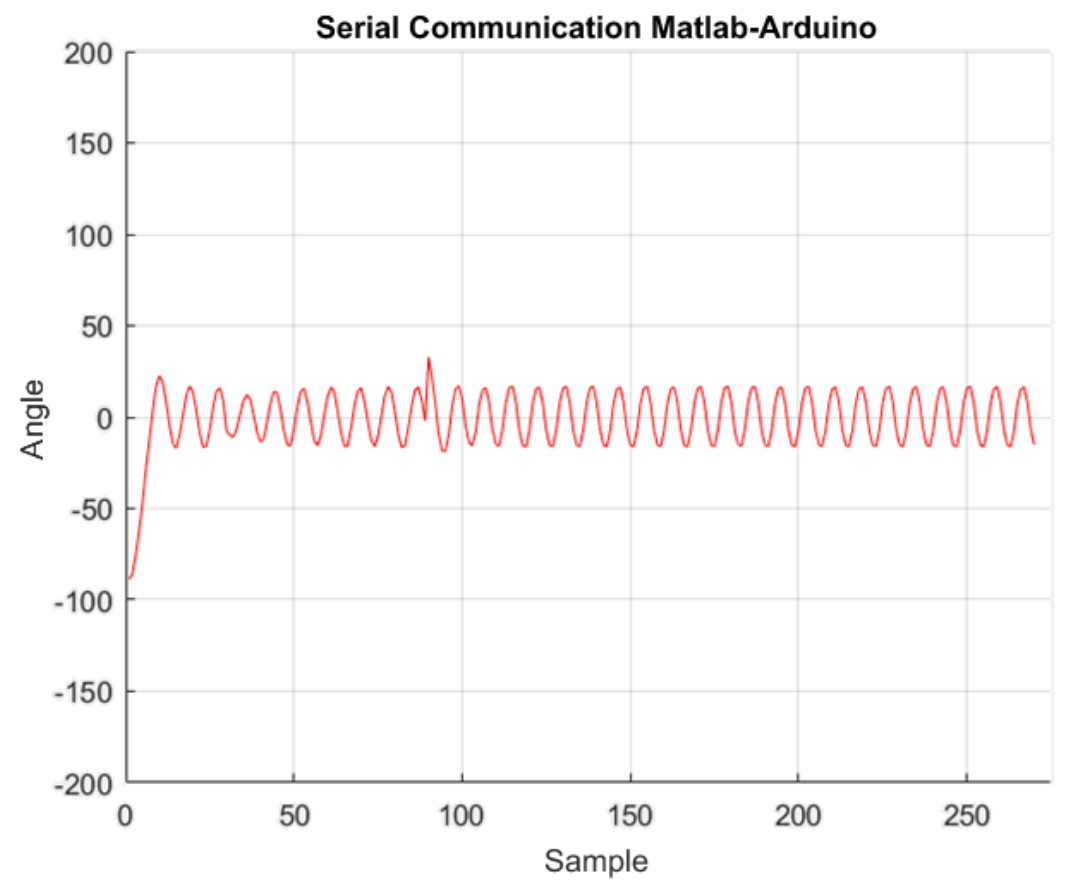

Fig. 3. System sustained oscillations.

The continual oscillation is achieved with a gain of 9.85 , this value is the critical gain, and the period of the oscillations is the critical period with a value of 0.55 seconds.

Now applying the Ziegler-Nichols tuning formulas to get the gains, there are 3 different alternatives: classic, with little overshoot and no overshoot at all like is shown in Table 2.

Table 2. Ziegler and Nichols tuning results.

\begin{tabular}{lccl}
\hline Controller & $k_{p}$ & $\tau_{i}$ & $\tau_{d}$ \\
\hline PID classic & $0.59 K_{c r}$ & $0.50 P_{c r}$ & $0.125 P_{c r}$ \\
\hline PID controlled overshot & $0.33 K_{c r}$ & $0.50 P_{c r}$ & $0.33 P_{c r}$ \\
\hline PID no overshot & $0.20 K_{c r}$ & $0.50 P_{c r}$ & $0.33 P_{c r}$ \\
\hline
\end{tabular}

Once $\mathrm{s} k_{p}, \tau_{i}$ and $\tau_{d}$ are calculated, then are entered to the controller to verify its result.

\subsection{Genetic Algorithm}

The Genetic Algorithm (GA), methodology implemented to the Control is the following explained: 
To initialize the GA is necessary to give some important information, the values used are show in Table 3 .

Table 3. Initial Conditions.

\begin{tabular}{ll}
\hline Initial condition & Value \\
\hline Seed & 3 \\
\hline Population size & 20 individuals \\
\hline Genes number & 3 \\
\hline Alleles number & 4 \\
\hline Tournament size & 3 \\
\hline Generations & 100 \\
\hline Mutation percentage & $10 \%$ \\
\hline Mutation numbers & 600 \\
\hline Mutations per generation & 6 \\
\hline Serial communication velocity & 11500 bauds \\
\hline
\end{tabular}

Then using the data obtain with Ziegler and Nichols tune, was propose the restriction to obtain the value for $k_{p}, \tau_{i}$ and $\tau_{d}$ variables avoiding making unstable the response, those values are show in Table 4.

Table 4. PID values.

\begin{tabular}{cccc}
\hline \multicolumn{4}{c}{ Gains } \\
\hline Kp & \multicolumn{2}{c}{ Ti } \\
\hline Resolution & 220 & Resolution & 2200 \\
\hline Max. Value & 4.6546 & Max. Value & 0.46546 \\
\hline Min. Value & 0.00454 & Min. Value & 0.000454 \\
\hline
\end{tabular}

The initial population was randomly initialized; the individuals have a structure as show in equation 4 :

$$
\operatorname{pobl}(1,1)=\{0100101101,1010101100,1101001010\}
$$

Objective function is generated following the algorithm 1 that describes the experiments to realize for measuring the behavior of each individual. 


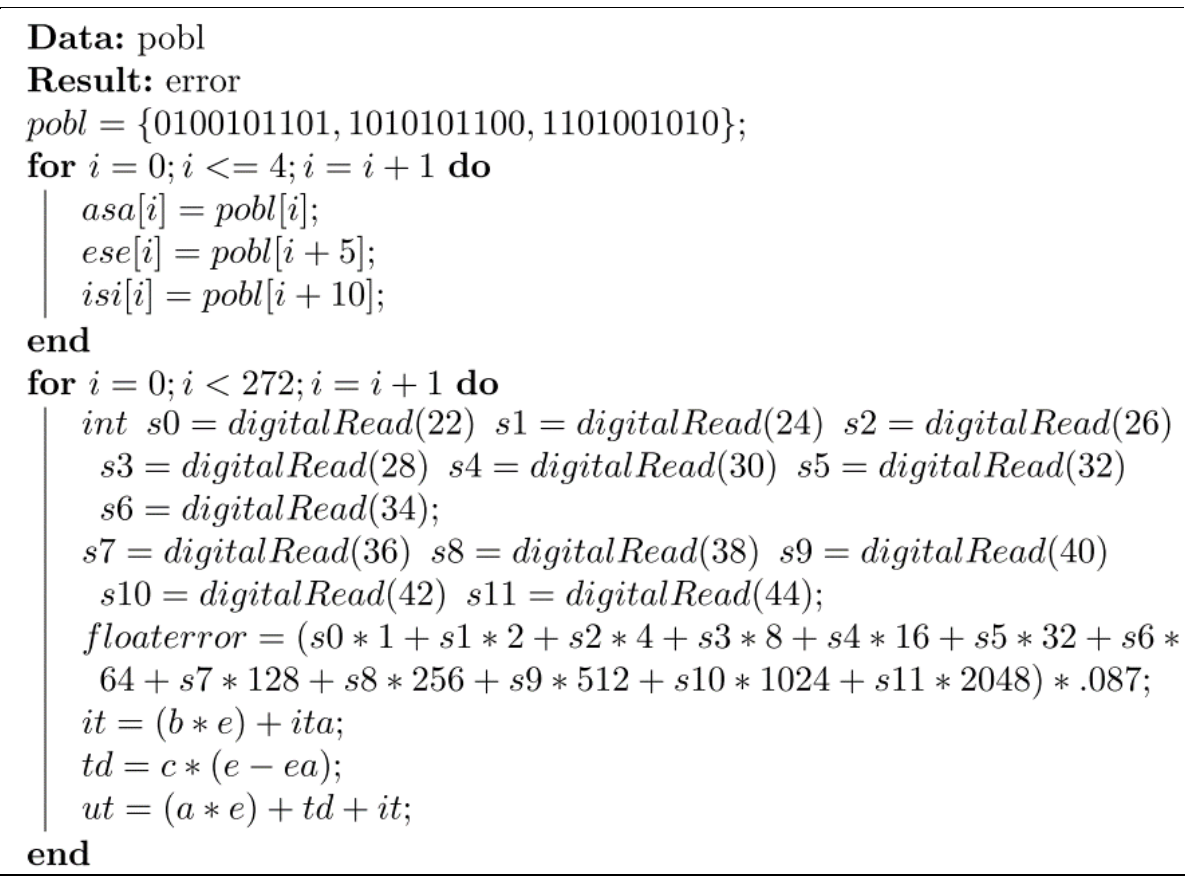

Algorithm 1. Fitness evaluation algorithm.

With the evaluated individuals the proposed algorithm is applied with the wellknown conventional operations, Fig. 4 shows the tournament flow diagram that apply for the selection process.

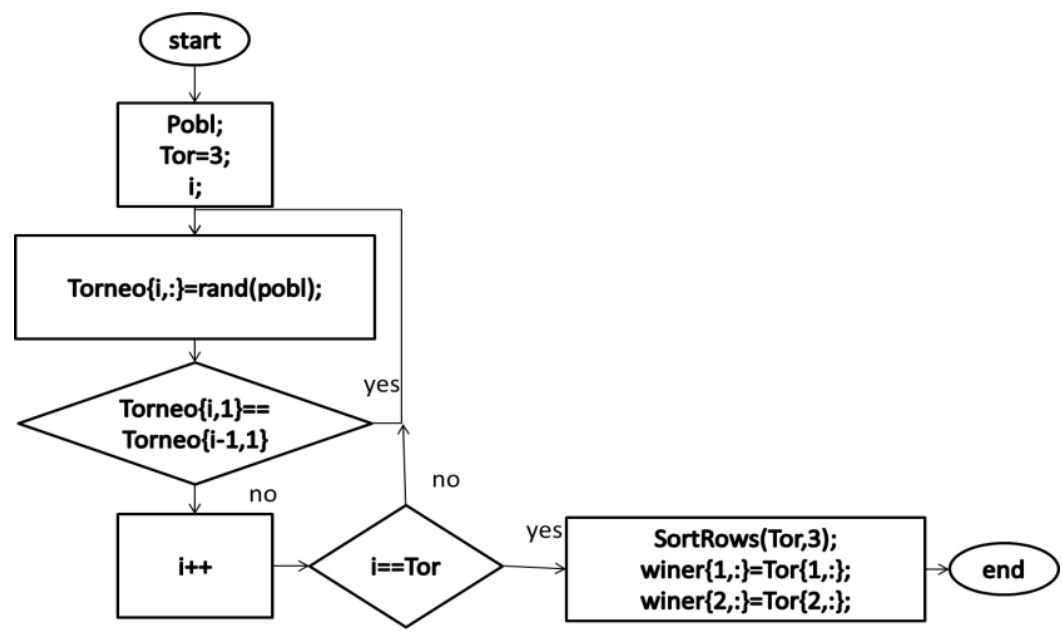

Fig. 4. Tournament flow diagram for selection process. 
For the crossover and mutation process the usual algorithm was applied having one crossover point and a mutation in only one allele by gene.

The winner of all the process gives the parameters (P, I, D), which are implemented in the PID controller.

\section{$5 \quad$ Results}

First was apply the classic PID controller and was plot the step response that appear in Fig. 5, where de PID variable values was $k_{p}=5.815, \tau_{i}=0.275$ and $\tau_{d}=0.068$. As it is shown the response have a continue oscillation approaching the reference value.

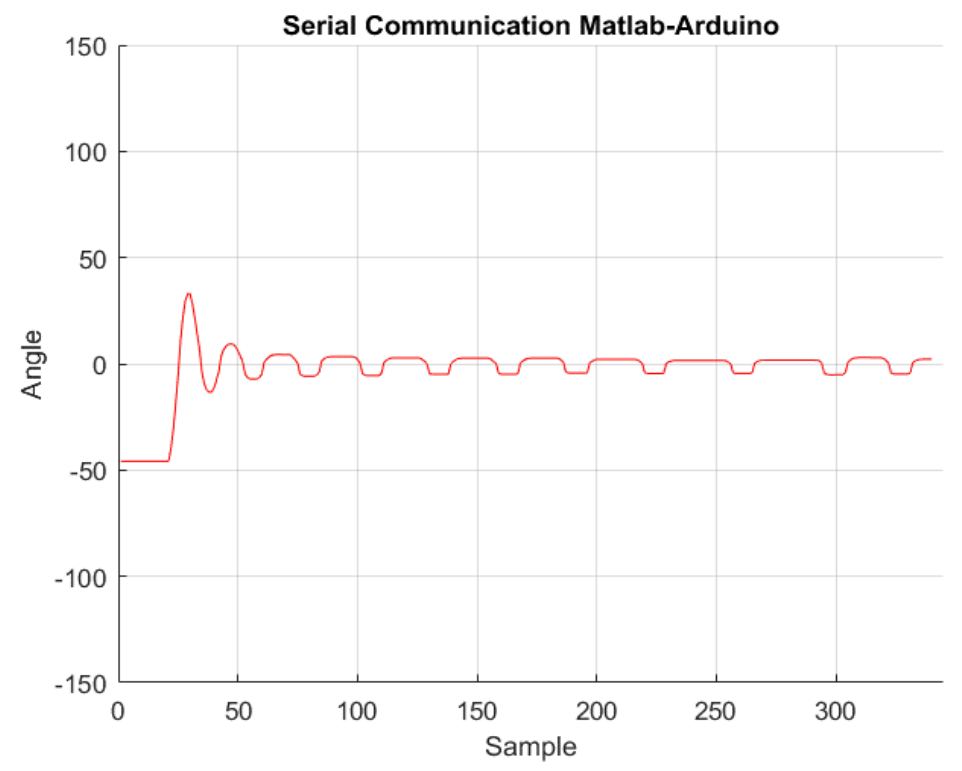

Fig. 5. Step response classic PID tune.

Then the process for controller gains with no overshooting was applied in a similar experiment to obtain the step response show in Fig. 6, in this case the establishment time greater than in other cases but the oscillation do not disappear, in this case the PID variable values are $k_{p}=1.97, \tau_{i}=0.275$ and $\tau_{d}=0.1815$.

However, it was not possible to perform the tuning by this method, since the system oscillated in all the cases. The overshooting case was omitted, because as is know if the overshoot is increase the system oscillates. Unfortunately, the Ziegler-Nichols method of critical earnings cannot present results for all control cases. 
Maximiliano Hernández García Rojas, Humberto Velasco Arellano, David Ubach González, et al.

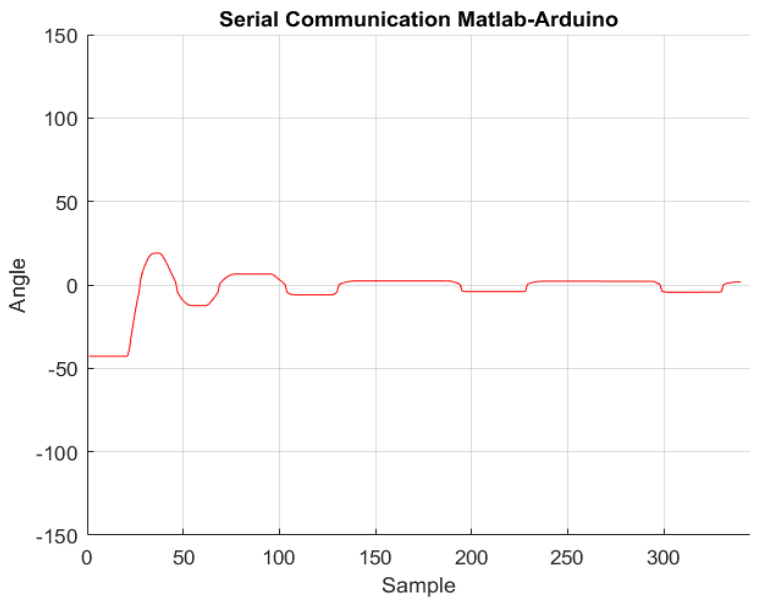

Fig. 6. Step response no overshooting PID tune.

To prove the functionality, there are three height parameters predetermined as height desired where the GA Control achieve this with the next voltage according to the set point.

After the genetic algorithm tunes the PID parameters the obtained results for PID control are given as $k_{p}=38.34, \tau_{i}=0.6575$ and $\tau_{d}=4.2725$, showing a behavior faster than the Ziegler and Nichols tune for all three cases, Fig. 7 shows the step response where it has an overshooting bigger than other controllers but have no oscillation approaching the reference.

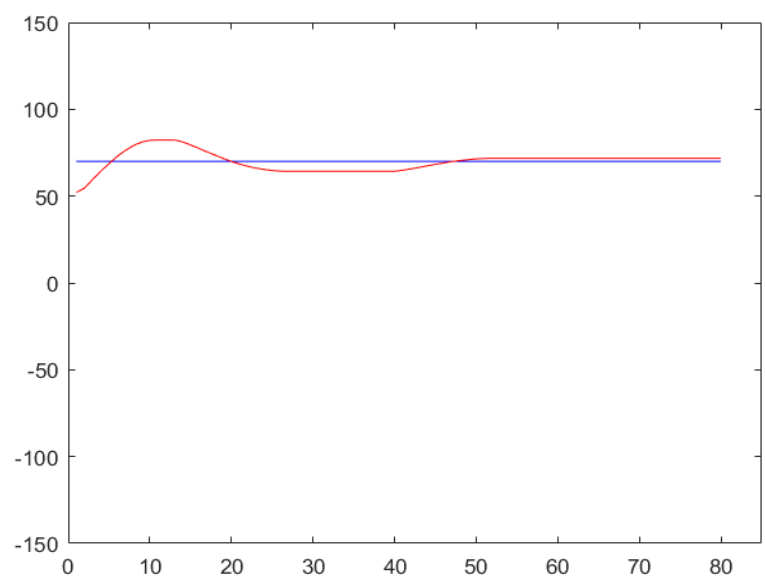

Fig. 7. PID response with GA tuning. 
To get this results the GA has an evolution represented by the optimization of the population, Fig. 8 shows this performance for the tuning process, the best element on the blue line have an evolution in the first generations and find the best local result, on the other hand the diversity of the population disappears then of the 350 generations, that prove that our algorithm has enough diversity and the result is not a lake suboptimal.

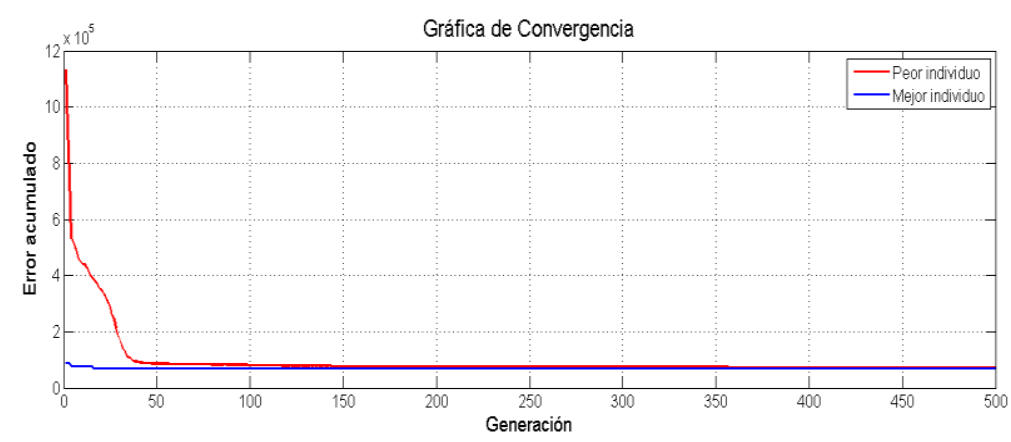

Fig. 8. GA population evolution.

\section{Conclusion}

This paper presents a comparison between PID controllers tuned with Ziegler and Nichols method and a using a Genetic Algorithms in a SCW system that takes as input the required orientation for the vehicle which is computed according to the detected lines, this paper shows that it is possible to obtain a self-tuning PID controller with better results than classical tuning techniques for a SWC assistant system.

The study of this control gives as a result information about the use of GA to tune a PID controller, showing the comparison between a well-known method that is Ziegler and Nichols tune, both have the capability to get a controller without knowledge about the system but, de GA have a better performance.

The PID has the capability to evolve and tune by itself the gains, was important to delimit the universe with the possible real values, to avoid get a lot of individuals that do not present an important behavior in the system.

The experiments need more time to develop and use a bigger population in more generations, to get a response with a better behavior.

In the future work, the controller will modify the reference of the warning get from the LDW to make an autonomous decision in an ADAS, raising the security conduction and performance. It is important to compare the actual control results with other optimization metaheuristic like PSO algorithms to find which the most proper tuning method is. 


\section{References}

1. Akatsuka, H., Kataoka, M., Matsumoto, T., Iida, H.: Electric power steering system with motor controller. pp. 933-971 (2016)

2. Aly, A.A.: Pid parameters optimization using genetic algorithm technique for electrohydraulic servo control system. Intelligent Control and Automation, 2(02), pp. 69 (2011)

3. Behensky, M.L., Moncrief, R.L., Durfey, E.J., Loper III, M.H.: Control device such as a steering wheel for video vehicle simulator with realistic feedback forces (1991)

4. Dobres, J., Reimer, B., Chahine, N.: The Effect of Font Weight and Rendering System on Glance-Based Text Legibility. Proceedings of the $8^{\text {th }}$ International Conference on Automotive User Interfaces and Interactive Vehicle Applications (2016)

5. Coughanowr, D.R., Koppel, L.B., et al.: Process systems analysis and control, 3 (1965)

6. Cuautle, T.E., Duarte, M.A., Reyes, C.A., Reyes, G.: Automatic synthesis of electronic circuits using genetic algorithms. Computación y Sistemas, 10(3) pp. 217-229 (2007)

7. Dahmani, H., Chadli, M., Rabhi, A., Hajjaji, A.: Vehicle dynamics and road curvature estimation for lane departure warning system using robust fuzzy observers: experimental validation. Vehicle Syst Dyn, 53(8), pp. 1135-1149 (2015)

8. Ezeta, R.F.d.B.: Análisis y diseño de sistemas de control digital. McGraw-Hill Interamericana (2013)

9. Flores, F., Aguilar, C., Suárez, M. S., Cuevas de la Rosa, F.: An identification genetic algorithm for a family of Duffing's system. Computación y Sistemas, 7(2), pp. 102-112 (2003)

10. Gaikwad, V., Lokhande, S.: Lane departure identification for advanced driver assistance. Ieee T Intell Transp 16(2), pp. 910-918 (2015)

11. Handy, J.P.: Steering-wheel control. US Patent 1,132,771(1915)

12. Hickman, J., Guo, F., Camden, M., Hanowski, R., Medina, A., Mabry, J.: Efficacy of roll stability control and lane departure warning systems using carrier-collected data. J Safety Res 52, pp. 59-63 (2015)

13. Jones, M.B.: Steering wheel control attachment apparatus. US Patent 5,520,071 ( 1996)

14. Kortli, Y., Marzougui, M., Atri, M.: Efficient implementation of a real-time lane departure warning system. pp. 1-6 (2016)

15. Li, W., Gong, X., Wang, Y., Liu, P.: A lane marking detection and tracking algorithm based on sub-regions. pp. 68-73 (2014)

16. Mahajan, R., Patil, A.: Lane departure warning system. International Journal of Engineering Education (2015)

17. Mejía, J.F.G., Sanchez, J.C.S., Fuentes, A.A.F., Martínez, J.A.P., Reyes, C.E.T.: Sintonización de un controlador proporcional-integral derivativo aplicado a una celda termoeléctrica: Una comparación entre algoritmos genéticos. Research in Computing Science 94, pp. 179$192(2015)$

18. Momani, S., Abo-Hammour, Z.S., Alsmadi, O.M.: Solution of inverse kinematics problem using genetic algorithms. Applied Mathematics \& Information Sciences 10(1), pp. 225 (2016)

19. Ogata, K.: Sistemas de control en tiempo discreto. Pearson educación (1996)

20. Prospero, L.A.C., Mejía-Lavalle, M., Ascencio, J.R., Rincón, V.V.V.: Aprendizaje incremental basado en población como buena alternativa al uso de algoritmos genéticos. Research in Computing Science 116, pp. 51-64 (2016)

21. Reagan, I., Anne, M.: Observed activation status of lane departure warning and forward collision warning of Honda vehicles at dealership service centers. Traffic Inj Prev, 17(8) (2016) 
22. L.J.: A genetic algorithm application for individual and group multicriteria decision making. Computación y Sistemas (2000)

23. Son, J., Yoo, H., Kim, S., Sohn, K.: Real-time illumination invariant lane detection for lane departure warning system. Expert Syst Appl 42(4), 1816-1824 (2015)

Sternlund, S., Strandroth, J., Rizzi, M., Lie, A., Tingvall, C.: The effectiveness of lane departure warning systems - A reduction in real-world passenger car injury crashes (2016)

24. Weise, T.: Global optimization algorithms theory and application (2009) 